Vietnam Journal of Mechanics, VAST, Vol. 28, No. 2 (2006), pp. 103-110

\title{
BUCKLING OF THE INITIAL IMPERFECT RECTANGULAR THIN PLATE WITH VARIABLE THICKNESS
}

\author{
Nguyen Thi Hien Luong and Dang Thuy Minh Tuong \\ Department of Civil Engineering, University of Technology of Ho Chi Minh City
}

\begin{abstract}
This paper analyzes the stability of the rectangular thin plate with sinusoidal changes in the plate thickness combined with initial curvature based on the large deflection theory. The buckling load for simply supported plates is defined using the energy method. The influence of the thickness variation parameter and the initial curvature parameter on the critical loads is investigated.
\end{abstract}

\section{INTRODUCTION}

Plates and shells as structural elements are seldom perfectly flat and of uniform thickness and the amount of initial curvature and variable thickness can affect the load-carrying capacity of structures. Recently, the problem of the influence of the thickness variation and the initial curvature on the buckling load has been researched by several authors, such as Timoshenko [1], Elishakoff et al [2], Zhiming [3], Yeh et al [4], Mateus et al [5], Nguyen and Tran [6], Ciancio [7].

In this paper, based on the theory of plates of large deflection, the stability study of imperfect rectangular thin plates with initial curvature and variable thickness is a major object. The energy method is used to determine the critical load factor of plates with variable thickness combined with initial deflection. The influence of the thickness non-uniformity parameter and the initial curvature parameter to the buckling load is investigated. General asymptotic formulae for the buckling load are derived and numerical results are investigated for compressive plates with the simply supported boundary condition (Fig. 1).

\section{ENERGY APPROACH}

This section aims at the study of the combined effect of thickness variation and initial imperfection on the buckling behavior of the rectangular thin plate. The simplest approach to the problem is a direct discussion of the energy criterion of elastic stability by means of the second variation of the potential energy. The energy method permits us to determine the buckling load of imperfect plates with variable thickness, as illustrated in [2]. Here, we consider the small thickness variation, and as a first approximation, only the terms up to the first order of thickness variation parameter are retained. The final product of this discussion is an equation that relates the variation parameter and the initial imperfection amplitude to the buckling load factor for the plate.

We assume that the displacement in the fundamental state is $\underline{u}_{0}\left(0,0, w_{0}\right)$.

The initial deflection is defined as

$$
w_{0}=-\mu h_{0} \sin \left(\frac{q \pi x}{a}\right),
$$




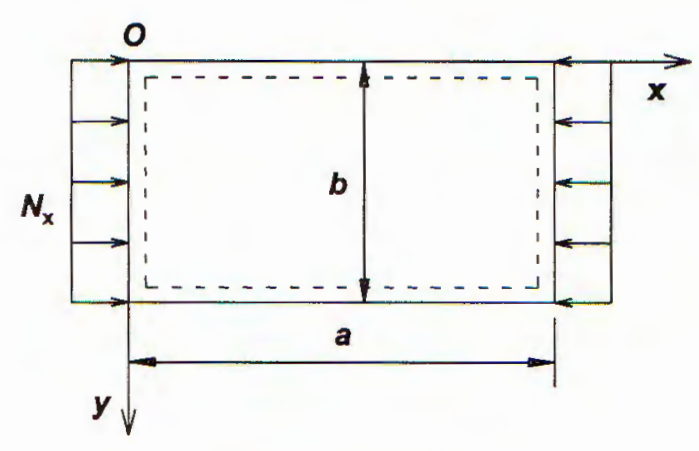

Fig. 1. Uniaxially compressed rectangular plate

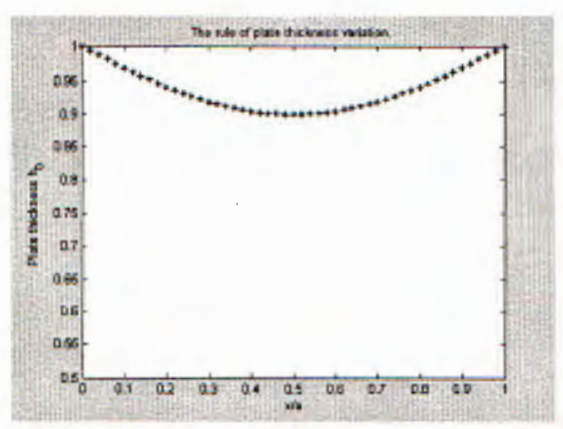

Fig. 2. Expression graph of thickness variation $h(x)$ when $\varepsilon=0.1$

with $\mu$ is the non-dimensional parameter describing the magnitude of the imperfection; $q$ is the wave number of the initial curvature function.

We assume that the plate thickness $h$ here is varying with sine function in $x$ direction:

$$
h(x)=h_{0}\left(1-\varepsilon \sin \frac{p \pi x}{a}\right), \quad \varepsilon \geq 0
$$

where $h_{0}$ is the plate constant thickness and $\varepsilon, p$ are the non-dimensional parameters indicating the magnitude and wave of the thickness variation, respectively. When $x=0$ and $x=a$, one has $h(x)=h_{0}$, for the case $x=a / 2$ : one has $h(x)=h_{0}(1-\varepsilon)$ (Fig. 2). The thickness parameter $\varepsilon$ is positive in order to achieve a detrimental effect by a "thinning" of the wall thickness. The parameters $\varepsilon, \mu$ vary from zero to 0.2 .

A deflection from the fundamental pre-buckling state is described by $\underline{u}(u, v, w)$.

Membrane strain energy of the rectangular thin plate is:

$$
\begin{aligned}
U_{m} & =\frac{1}{2} \int_{0}^{b} \int_{0}^{a}\left(N_{x} \varepsilon_{x}+N_{y} \varepsilon_{y}+N_{x y} \gamma_{x y}\right) d x d y= \\
& =\frac{E}{2\left(1-\nu^{2}\right)} \int_{0}^{b} \int_{0}^{a} h\left(\varepsilon_{x}^{2}+\varepsilon_{y}^{2}+2 \nu \varepsilon_{x} \varepsilon_{y}+\frac{1}{2}(1-\nu) \gamma_{x y}^{2}\right) d x d y .
\end{aligned}
$$

Bending strain energy reads

$$
U_{b}=\frac{1}{2} \int_{0}^{b} \int_{0}^{a} D(x, y)\left\{\left(\frac{\partial^{2} w}{\partial x^{2}}+\frac{\partial^{2} w}{\partial y^{2}}\right)^{2}-2(1-\nu)\left[\frac{\partial^{2} w}{\partial x^{2}} \frac{\partial^{2} w}{\partial y^{2}}-\left(\frac{\partial^{2} w}{\partial x \partial y}\right)^{2}\right]\right\} d x d y
$$

For the rectangular thin plate under compression load $N$, the potential energy of the applied load takes the form

$$
\Omega=-\frac{1}{2} \int_{0}^{b} \int_{0}^{a} N\left(\frac{\partial w}{\partial x}+\frac{\partial w_{0}}{\partial x}\right)^{2} d x d y
$$


where $w_{0}$ is the geometric initial imperfection.

Thus, the total potential energy reads

$$
\Pi=U_{m}+U_{b}+\Omega
$$

In the case of large deflection, the strain - displacement increments relations are of the forms [1], [2]:

$$
\varepsilon_{x}=\frac{\partial u}{\partial x}+\frac{1}{2}\left(\frac{\partial w}{\partial x}\right)^{2} ; \quad \varepsilon_{y}=\frac{\partial v}{\partial y}+\frac{1}{2}\left(\frac{\partial w}{\partial y}\right)^{2} ; \quad \gamma_{x y}=\frac{\partial u}{\partial y}+\frac{\partial v}{\partial x}+\frac{\partial w}{\partial x} \frac{\partial w}{\partial y}
$$

where $u, v$ are the displacements in $x$ and $y$ directions, $w$ is the deflection, positive outward; $\varepsilon_{x}, \varepsilon_{y}$ and $\gamma_{x y}$ are strain components.

Substituting Eq. (2.7) into Eq. (2.3) and Eqs. (2.3) - (2.5) into Eq. (2.6), we obtain the energy expression in the general anisotropic case:

$$
\begin{aligned}
\Pi= & \frac{1}{2} \int_{0}^{b} \int_{0}^{a}\left\{A_{11}\left[\frac{\partial u}{\partial x}+\frac{1}{2}\left(\frac{\partial w}{\partial x}\right)^{2}\right]^{2}+2 A_{12}\left[\frac{\partial u}{\partial x}+\frac{1}{2}\left(\frac{\partial w}{\partial x}\right)^{2}\right]\left[\frac{\partial v}{\partial y}+\frac{1}{2}\left(\frac{\partial w}{\partial y}\right)^{2}\right]+\right. \\
& +A_{22}\left[\frac{\partial v}{\partial y}+\frac{1}{2}\left(\frac{\partial w}{\partial y}\right)^{2}\right]^{2}+A_{66}\left[\frac{\partial u}{\partial y}+\frac{\partial v}{\partial x}+\frac{\partial w}{\partial x} \frac{\partial w}{\partial y}\right]^{2}+D_{11}\left(\frac{\partial^{2} w}{\partial x^{2}}\right)^{2}+ \\
& \left.+2 D_{12} \frac{\partial^{2} w}{\partial x^{2}} \frac{\partial^{2} w}{\partial y^{2}}+D_{22}\left(\frac{\partial^{2} w}{\partial y^{2}}\right)^{2}+D_{66}\left(\frac{\partial^{2} w}{\partial x \partial y}\right)^{2}-N\left(\frac{\partial w}{\partial x}+\frac{\partial w_{0}}{\partial x}\right)^{2}\right\} d x d y
\end{aligned}
$$

In the isotropic case, expression (2.8) is the total potential energy with:

$$
\begin{aligned}
& A_{11}=A_{22}=\frac{E h}{1-\nu^{2}} ; \quad A_{12}=\nu A_{11} ; \quad A_{66}=\frac{1-\nu}{2} A_{11} ; \quad A_{16}=A_{26}=0, \\
& D_{11}=D_{22}=\frac{E h^{3}}{12\left(1-\nu^{2}\right)} ; \quad D_{12}=\nu D_{11} ; \quad D_{66}=2(1-\nu) D_{11} ; \quad D_{16}=D_{26}=0,
\end{aligned}
$$

and $\nu$ is Poisson's ratio, $E$ is the modulus of elasticity.

The energy variation is performed at the fundamental pre-buckling state ([2]). Rejecting the fourth variation of the potential energy from expression (2.8), one obtains:

$$
\Pi=P_{11}\left[u_{0}, u\right]+P_{2}[u]+P_{3}[u],
$$

where the bilinear term $P_{11}\left[u_{0}, u\right]$ due to the geometric initial imperfection is given by:

$$
P_{11}\left[u_{0}, u\right]=-N^{c r} \int_{0}^{b} \int_{0}^{a} \frac{\partial w}{\partial x} \frac{d w_{0}}{d x} d x d y .
$$



modes:

In expression (2.9), $P_{2}[u]$ is the second (quadratic) variation of the energy for buckling

$$
\begin{aligned}
P_{2}[u] & =\frac{1}{2} \int_{0}^{b} \int_{0}^{a}\left[A_{11}\left(\frac{\partial u}{\partial x}\right)^{2}+2 A_{12} \frac{\partial u}{\partial x} \frac{\partial v}{\partial y}+A_{22}\left(\frac{\partial v}{\partial y}\right)^{2}+\right. \\
& +A_{66}\left(\frac{\partial u}{\partial y}+\frac{\partial v}{\partial x}\right)^{2}+D_{11}\left(\frac{\partial^{2} w}{\partial x^{2}}\right)^{2}+2 D_{12} \frac{\partial^{2} w}{\partial x^{2}} \frac{\partial^{2} w}{\partial y^{2}}+ \\
& \left.+D_{22}\left(\frac{\partial^{2} w}{\partial y^{2}}\right)^{2}+D_{66}\left(\frac{\partial^{2} w}{\partial x \partial y}\right)^{2}-N^{c r}\left(\frac{\partial w}{\partial x}\right)^{2}\right] d x d y
\end{aligned}
$$

and $P_{3}[u]$ is the third variation of the energy:

$$
\begin{aligned}
& P_{3}[u]=\frac{1}{2} \int_{0}^{b} \int_{0}^{a}\left\{A_{11} \frac{\partial u}{\partial x}\left(\frac{\partial w}{\partial x}\right)^{2}+A_{12}\left[\frac{\partial u}{\partial x}\left(\frac{\partial w}{\partial y}\right)^{2}+\frac{\partial v}{\partial y}\left(\frac{\partial w}{\partial x}\right)^{2}\right]\right. \\
& \left.+A_{22} \frac{\partial v}{\partial y}\left(\frac{\partial w}{\partial y}\right)^{2}+2 A_{66}\left(\frac{\partial u}{\partial y}+\frac{\partial v}{\partial x}\right) \frac{\partial w}{\partial x} \frac{\partial w}{\partial y}\right\} d x d y
\end{aligned}
$$

If the rectangular plate is simply supported around the periphery and edges are immovable in the plane of plate, then the boundary conditions are:

$$
\begin{aligned}
w=w_{x x x}=w_{, y y} & =0 \quad \text { at } \quad x=0, a ; \quad y=0, b \\
u & =0 \quad \text { at } \quad x=0, \frac{a}{2}, a ; \quad y=0, b \\
v & =0 \quad \text { at } \quad x=0, a ; \quad y=0, \frac{b}{2}, b .
\end{aligned}
$$

In applying the energy method, we must assume suitable expressions for the displacements $u, v, w$. The proposed displacement functions chosen for this case are given by the following series:

$$
\begin{aligned}
& w=b_{0} h_{0} \sin \left(\frac{q \pi x}{a}\right)+C_{m} h_{0} \sin \left(\frac{m \pi x}{a}\right) \sin \left(\frac{n \pi y}{b}\right), \\
& u=Q_{m} C_{m} h_{0} \sin \left(\frac{2 m \pi x}{a}\right) \sin \left(\frac{n \pi y}{b}\right), \\
& v=K_{m} C_{m} h_{0} \sin \left(\frac{m \pi x}{a}\right) \sin \left(\frac{2 n \pi y}{b}\right),
\end{aligned}
$$

where $b_{0}, K_{m}, C_{m}$ and $Q_{m}$ are constants; $m, n$ are the wave numbers of the buckling mode in the $x$ and $y$ directions of thin plate, $m, n, q=1,3,5, \ldots$

From the condition of minimum of total energy, one obtains:

$$
\begin{aligned}
& \frac{\partial \Pi}{\partial b_{0}}=0, \\
& \frac{\partial \Pi}{\partial C_{m}}=0,
\end{aligned}
$$




$$
\begin{aligned}
\frac{\partial \Pi}{\partial Q_{m}} & =0, \\
\frac{\partial \Pi}{\partial K_{m}} & =0 .
\end{aligned}
$$

Consider the following normalization:

$$
\lambda=\frac{N^{c r}}{N_{0}^{c r}}
$$

where $\lambda$ is the non-dimensional buckling load factor, $N_{0}^{c r}$ is the buckling load of the rectangular plate with $\mu=0, \varepsilon=0 ; N^{c r}$ is the buckling load of the rectangular thin plate with the variable thickness and the initial curvature $\varepsilon \neq 0, \mu \neq 0$.

Substituting the solution $C_{m}=0$ of equation (2.16) into equation (2.15), we determine $b_{0}$. If $C_{m} \neq 0$, from equation (2.17) and (2.18), we can determine $Q_{m}$ and $K_{m}$.

Substitution $b_{0}, K_{m}, Q_{m}$ into equation (2.16) leads to the equation expressed the relation between the initial curvature amplitude $\mu$, the variable thickness parameter $\varepsilon$ and the buckling load factor $\lambda$ :

$$
F(\mu, \varepsilon, \lambda)=0 .
$$

Solving the equation (2.30), one can determine the buckling load factor $\lambda_{\min }$.

\section{DETERMINATION OF THE BUCKLING LOAD FACTOR}

When the rectangular plate is simply supported, the number of sine-half-waves in series functions can be chosen as: $m=n=p=1, q=3$ and we obtain: $w_{0}=-\mu h_{0} \sin \left(\frac{3 \pi x}{a}\right)$ and $h(x)=h_{0}\left(1-\varepsilon \sin \frac{\pi x}{a}\right)$

$$
\begin{aligned}
& w=b_{0} h_{0} \sin \left(\frac{3 \pi x}{a}\right)+C_{m} h_{0} \sin \left(\frac{\pi x}{a}\right) \sin \left(\frac{\pi y}{b}\right), \\
& u=Q_{m} C_{m} h_{0} \sin \left(\frac{2 \pi x}{a}\right) \sin \left(\frac{\pi y}{b}\right), \\
& v=K_{m} C_{m} h_{0} \sin \left(\frac{\pi x}{a}\right) \sin \left(\frac{2 \pi y}{b}\right) .
\end{aligned}
$$

In the case of square plate: $r=\frac{a}{b}=1$ and $\nu=0.3$, substituting Eqs. (2.10)- (2.12) into the second and third variations, we obtain, after retaining only the first-order terms in $\varepsilon$,

$$
P_{11}\left[u_{0}, u\right]=\frac{9}{2} \pi^{2} b_{0} h_{0}^{2} \mu N^{c r},
$$




$$
\begin{aligned}
P_{2}[u]= & \left(\frac{435}{728} \pi^{2} E h_{0}^{3} K_{m}^{2}+\frac{25}{546 b^{2}} \pi^{4} E h_{0}^{5}-\frac{835}{546} \pi E h_{0}^{3} K_{m}^{2} \varepsilon-\frac{44}{39} \pi E h_{0}^{3} Q_{m}^{2} \varepsilon-\right. \\
& -\frac{95}{273} \pi E h_{0}^{3} K_{m} Q_{m} \varepsilon+\frac{435}{728} \pi^{2} E h_{0}^{3} Q_{m}^{2}-\frac{55}{182 b^{2}} \pi^{3} E h_{0}^{5} \varepsilon+\frac{80}{63} E h_{0}^{3} K_{m} Q_{m}- \\
& \left.-\frac{1}{8} \pi^{2} h_{0}^{2} N^{c r}\right) C_{m}^{2}+\left(\frac{675}{364 b^{2}} \pi^{4} E h_{0}^{5}-\frac{9}{4} \pi^{2} h_{0}^{2} N^{c r}-\frac{7290}{637 b^{2}} \pi^{3} E h_{0}^{5} \varepsilon\right) b_{0}^{2}, \\
P_{3}[u]= & \frac{\pi E h_{0}^{4} C_{m}^{2} b_{0}}{183456}\left(124740 \pi^{2} Q_{m}+5040 \pi K_{m} \varepsilon-100224 \pi Q_{m} \varepsilon-16128 K_{m}\right) .
\end{aligned}
$$

From Eqs. (2.15), (2.16), (2.17) and (2.18), one obtains:

$$
\begin{gathered}
b_{0}=-\frac{637 \mu b^{2} N^{c r}}{525 \pi^{2} E h_{0}^{3}-637 b^{2} N^{c r}-3240 \pi E h_{0}^{3} \varepsilon}, \\
K_{m}=-\frac{108 \pi^{2} h_{0} b_{0}\left(187425 \pi^{2} \varepsilon-673680 \pi+620544 \varepsilon-175360 \pi \varepsilon^{2}\right)}{35 b\left(14553648 \pi^{2} \varepsilon^{2}+1896960 \pi \varepsilon-3461120-13633596 \pi^{3} \varepsilon+3065445 \pi^{4}\right)}, \\
Q_{m}=-\frac{9 \pi h_{0} b_{0}\left(372736+5611056 \pi^{2} \varepsilon^{2}-218624 \pi \varepsilon-9123732 \pi^{3} \varepsilon+2713095 \pi^{4}\right)}{14 b\left(14553648 \pi^{2} \varepsilon^{2}+1896960 \pi \varepsilon-3461120-13633596 \pi^{3} \varepsilon+3065445 \pi^{4}\right)} .
\end{gathered}
$$

When $\varepsilon=0$ and $\mu=0, N_{0}^{c r}$ is determined as follows:

$$
N_{0}^{c r}=\frac{100}{273} \frac{\pi^{2} E h_{0}^{3}}{b^{2}} .
$$

We receive the same formula for a simply supported square plate with constant thickness and $\nu=0.3$ in [1] and [6]:

$$
N_{0}^{c r}=\frac{\pi^{2} D}{b^{2}}\left(\frac{a}{b}+\frac{b}{a}\right)^{2}=\frac{\pi^{2} D}{b^{2}}(1+1)^{2}=\frac{4 \pi^{2} E h_{0}^{3}}{12 b^{2}\left(1-\nu^{2}\right)}=\frac{100}{273} \frac{\pi^{2} E h_{0}^{3}}{b^{2}} .
$$

When $\varepsilon \neq 0$ and $\mu \neq 0$, we have the relation between $\lambda, \varepsilon$ and $\mu$ :

$$
\begin{aligned}
& -124.74 \varepsilon^{5}-115.82 \lambda \varepsilon^{4}+548.33 \varepsilon^{4}+452.12 \lambda \varepsilon^{3}+1.4413 \lambda^{2} \varepsilon^{3} \mu^{2}-889.88 \varepsilon^{3} \\
& -33.254 \lambda^{2} \varepsilon^{3}+113.2 \lambda^{2} \varepsilon^{2}-603.79 \lambda \varepsilon^{2}+667.55 \varepsilon^{2}-3.0394 \lambda^{3} \varepsilon^{2}-12.978 \lambda^{2} \varepsilon^{2} \mu^{2} \\
& +8.8189 \lambda^{3} \varepsilon+35.569 \lambda^{2} \varepsilon \mu^{2}+322.8 \lambda \varepsilon-235.28 \varepsilon-116.83 \lambda^{2} \varepsilon+31.617 \\
& -59.72 \lambda+34.349 \lambda^{2}-26.773 \lambda^{2} \mu^{2}-6.246 \lambda^{3}=0 .
\end{aligned}
$$

\section{ANALYSIS AND DISCUSSION}

In order to investigate the variation of the buckling load for the plate due to the small thickness variation and the initial curvature, the influence of the thickness parameter $\varepsilon$ and the initial curvature parameter $\mu$ is studied. The following figures are presented for square plates $(r=1)$ with $\nu=0.3$. 
From Eq. (3.5), the relationship between $\lambda$ and $\mu$ is shown in Fig. 3 for different values of $\varepsilon$, the relationship between $\lambda$ and $\varepsilon$ is shown in Fig. 4 for different values of $\mu$. The combined effect of the thickness variation and the initial imperfection on the buckling load is illustrated in Fig. 5.

The results obtained show that, when the initial imperfection is present, the combination of the initial imperfection and the thickness variation reduces the buckling load factor even more drastically. When the amplitude of the thickness variation and the initial imperfection amplitude are $\varepsilon=0.1, \mu=0.2$, the buckling load factor of plate is reduced by $28.2 \%$. If the effect of thickness variation is not considered, the buckling load factor of plate is reduced by $8.2 \%$ for $\mu=0.2$ from its counterpart of the case with constant thickness. In this case, when $\varepsilon=0.2, \mu=0.2$, the buckling load factor $\lambda$ decreases up to $48.5 \%$.

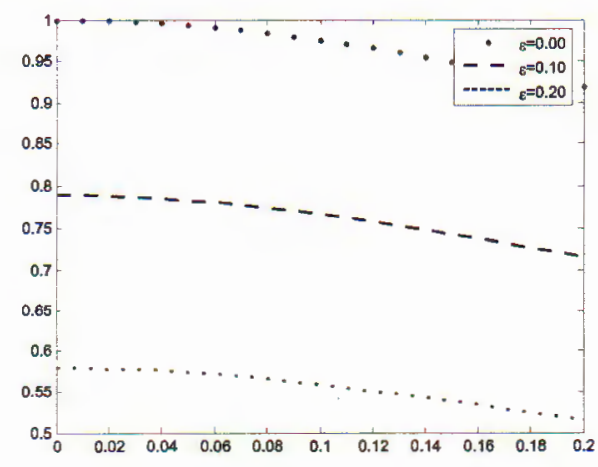

Fig. 3. Relation between $\lambda$ and $\mu$ for $\varepsilon=0,0.1,0.2$

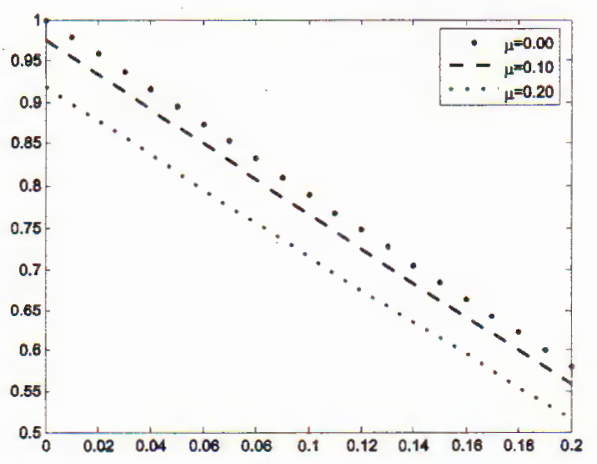

Fig. 4. Relation between $\lambda$ and $\varepsilon$ for $\mu=$ $0,0.1,0.2$

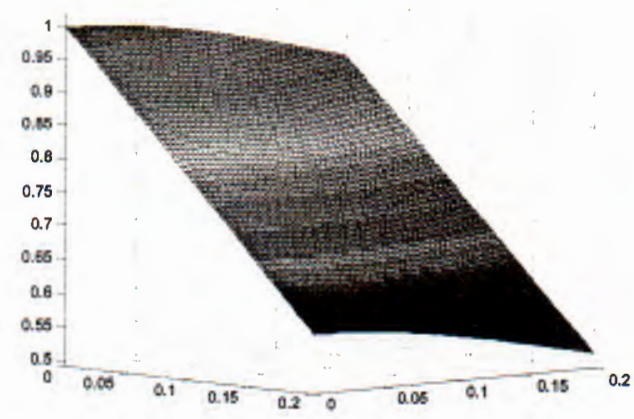

Fig.5. Relation between $\lambda, \varepsilon$ and $\mu$

\section{CONCLUSION}

In this paper, the energy expressions for imperfect plates have been given in the case of large deflection. Based on these expressions, a detailed study of the imperfect rectangular thin plate with thickness varying along the $x$-axes with sine functions has been presented. The formulae for the buckling load have been derived using the energy method. 
From the obtained results, one can conclude that the variable thickness and the initial curvature may cause a reduction of the load carrying capacity of plate structures, and so this effect should be taken into account in the design of plate structures.

This work was done under the support of Natural Science Council of Vietnam.

\section{REFERENCES}

1. S. P. Timoshenko, J. M. Gere, Theory of Elastic Stability, McGraw-Hill, 1990.

2. I. Elishakoff, Y. Li, J. M. Starners, Non-Classical Problem in the Theory of Elastic Stability. Cambridge University Press, 2001.

3. Ye Zhiming, Non-linear Analysis and Optimization of Shallow Shell of Variable Thickness, Mech. Res. Comm, 1997.

4. Yen-Liang Yeh, Cha'o-Kuang Chen, Hsin-Yi Lai, Chaotic and Bifurcation Dynamics for a Simply Supported Rectangular Plate of Thermo-Mechanical Coupling in Large Deflection, Chao's, Solution and Fractal's 13 (2002) 1493-1506.

5. A. F. Mateus, J. A. Wits, Post-Buckling of Corroded Steel Plates: A Comparative Analysis.

6. Nguyen T. Hien Luong, Tran H. Tri, Influence of variable thickness on stability of rectangular plate under compression, Mechanics Research Communications 32 (2005) (2) $139-146$.

7. P. M. Ciancio, Determination of Critical Loads in Annular Plate of Variable Thickness under Different Load Conditions, European Congress on Computational Methods in Applied Sciences and Engineering, Eccomas 2000, Barcelona (2000).

8. V. D. Cliusnhicov, Lectures on Stability of Deformable Solids, Publisher of Moscow University, 1986. (in Russian).

9. C. Y. Chia, Nonlinear Analysis of Plates. McGraw-Hill, N.Y., 1980.

10. D. T. Minh Tuong, Study of imperfect plate structures with a large deflection and variable thickness, Thesis of Master Dissertation in Civil Engineering, HCMC Polytechnic Institute of Technology, 2004.

Received October 28, 2005

Revised May 20, 2006

\section{ỔN DỊNH CỬ TÁM CHŨ̃ NHẬT KHÔNG HOÀN HẢO CÓ CHIỀU DÀY THAY DỔI THEO LÝ THUYẾT TẤM CÓ Dộ VÕNG LỚN}

Phân tích ồn định phi tuyến tấm chữ nhật có chiều dày thay đổi theo phương $\mathrm{x}$ với qui luật hình sin và có độ cong ban đầu dựa trên lý thuyết tấm mỏng có độ võng lớn. Lực tới hạn được xác định cho tấm tựa tự do bằng cách sử dụng phương pháp năng lượng. Ảnh hưởng của sự thay đổi chiều dày và thông số độ cong ban đầu đến hệ số tải trọng tới hạn được khảo sát. 